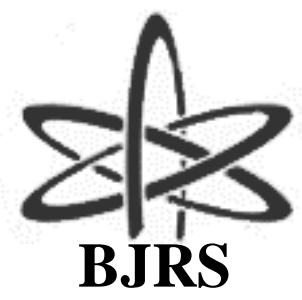

BRAZILIAN JOURNAL

$\mathrm{OF}$

RADIATION SCIENCES

07-03 (2019) 01-13

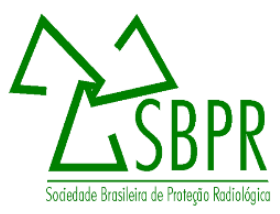

\title{
Total Workload for Radiotherapy Facilities with Volumetric Modulated Arc Treatment
}

\author{
Reis Junior ${ }^{\mathrm{a}}$ J.P., Alves ${ }^{\mathrm{b}}$ V.G. L., Fairbanks ${ }^{\mathrm{c}}$ L.R. \\ ${ }^{a}$ Ingá Radiotherapy Clinic/Department of Medical Physics, 24.210-470, Rio de Janeiro, Rio de Janeiro, Brazil \\ passosjjrj@hotmail.com \\ ${ }^{b}$ National Institute of Cancer, INCA, 20.230-130, Rio de Janeiro, Rio de Janeiro, Brazil \\ victorgabr@gmail.com \\ Américas Oncologia/Department of Medical Physics, 24.230-251, Niterói, Rio de Janeiro, Brazil \\ leandrofairbanks@americasoncologia.com.br
}

\begin{abstract}
In recent years, there have been major changes in radiotherapy, particularly in dose delivery for treatments using the techniques of Three-Dimensional Conformal Radiation Therapy (3D-CRT), Intensity-Modulated Radiation Therapy (IMRT) and Volumetric Modulated Arc Therapy (VMAT). However, in the literature, no workload results for radiation therapy treatments performed exclusively with the VMAT technique were found. In this study, a new workload and a new VMAT factor will be proposed. For such, patient data originating from management and planning systems were acquired, such as dose values, monitor units, numbers of arcs per patient and number of hypofractionated treatments. The average clinical workload values for conventional treatments were $328 \mathrm{~Gy} /$ week, resulting in a VMAT factor of 1.93; similarly, for hypofractionated treatments, the clinical workload was 33Gy/week and the VMAT factor was 1.54. The total workload has a value of 596 Gy/week, less than the value used in the facility shielding design, $1250 \mathrm{~Gy} /$ week, and the average value of VMAT factor for conventional and hypofractionated treatments showed that a smaller amount of $\mathrm{C}$ should be used at facilities that perform exclusive VMAT treatment.
\end{abstract}

Keywords: radiation protection, shielding, workload, VMAT. 


\section{INTRODUCTION}

In recent years, there have been drastic changes in the modalities of cancer treatment, particularly with regard to shielding calculation; the change from of conformal radiation therapy (3D CRT) to intensity modulated radiation therapy (IMRT) has resulted in the increase of monitor units. Rodgers 2011 reports that the IMRT factor - which is a comparison between the monitor units (MU) of a conventional treatment with the MU in IMRT treatment having the same dose - has values ranging from 2 to $10[1,2]$. Increasing this factor would thus result in an additional tenthvalue layer (TVL) in the shielding of radioactive facilities.

The theory of dose delivery through volumetric arcs was proposed by $\mathrm{Yu}$ [3] as intensity modulated arc therapy (IMAT). However, it was only possible to implement the proposed technology, volumetric modulated arc therapy (VMAT), through the development of optimization algorithms created for this new technique of treatment $[4,5]$. Oliver et al. [6] reported a shorter beam-on time, with a sharp decrease in the MU when comparing IMRT with VMAT.

This lower value of MU firmly impacts in clinical and physical workload, so this work aims to evaluate the new workloads with these technological innovations in patient treatment planning and quality assurance of medical accelerators.

Through the analysis of all patients treated with the VMAT technique for two years, it was possible to obtain a new VMAT factor, which is lower than what is recommended by the national regulatory agency.

\section{MATERIALS AND METHODS}

The total workload is a quantity of the great importance in radiation protection. It is defined as the total dose released by the linear accelerator (linac) in Gy/week or Gy/year at a well-defined point, usually at one meter from the source, at the isocenter of the linac. It is quantified by adding the clinical workloads (patient treatments) and the physical workload (quality control, acceptance and commissioning of the linac, preventive maintenance, and eventually in some research irradiations). 
To quantify this clinical workload over two years (2012 to 2014), the following data were extracted from the Aria $^{\mathrm{TM}}$ data management system (Varian Medical Systems, Inc.): data on total dose, number of arcs, number of monitor units MU's, anatomical sites, treatment stages, and number of hypofractionated treatments. In the physical workload, the MU's referring to commissioning and acceptance tests, preventive maintenance, quality control (daily, monthly, quarterly, semi-annual and annual) were quantified.

To obtain be total workload, some standardizations were made:

1 year $=50$ weeks.

1 month $=4$ weeks.

Total time of operation of the facility: 20 years or 1000 weeks.

\subsection{Clinical Workload}

In a period of two years, 953 patients were treated in the Americas Oncology Center with the VMAT technique $[3,7]$. The institution did not perform treatments of total body irradiation (TBI), total skin irradiation (TSI), or intraoperative radiation therapy (IORT).

For the clinical workload [8,9], conventional dose treatments were considered with fractionation ranging from 25 to 39 sessions at a prescribed dose below $3 \mathrm{~Gy} /$ day, with a total of 852 treatments $(89.4 \%)$. For the case of hypofractionation, the maximum number of sessions considered was 15 , with doses ranging from 3 to $20 \mathrm{~Gy}$. The total number of institutional hypofractionated treatments is 101 corresponding to $10.6 \%$ of all treatments.

\subsection{Clinical Workload per Site}

The values of dose and MU in radiotherapy planning vary greatly between different anatomical regions. For example, conventional dose values lie between 1.8 and $3 \mathrm{~Gy}$, however treatments of hypofractionation, stereotactic body radiotherapy SBRT of liver, lung and spine have dose values ranging between 6.2 and $18 \mathrm{~Gy}$ [10], and extracranial radiosurgery has values ranging from 12 to 20 Gy [11]. The dose values have medical evaluation and determinant influence in the literature [12], which, aside from the anatomical region, highlights lesion volume, proximity to an organ at risk 
(brainstem, spinal cord, esophagus, chiasm), immobilization accessories, and possibility to execute image-guided radiotherapy IGRT with cone beam, for instance.

\subsection{Institutional Physical Workload}

The physical workload encompasses any radiation that is not patient related, for example: commissioning $[13,14]$ acceptance, and all quality controls of the linac. In the latter, the recommendations of Task Group TG-142 were followed [15], and the MU values were considered, following the equivalence of $100 \mathrm{MU}=1 \mathrm{~Gy}$.

- Daily Quality Control (dosimetry constancy, all energies);

- Monthly Quality Control (dosimetry constancy, profile, dose rate);

- Quarterly Quality Control;

- Annual Quality Control [16] (absolute and relative dosimetry, beam profile, specification of beam quality, output factor, cone factors (electrons), filter factor, dose linearity, repeatability, dose rate dependency, depth dose percentage, $\mathrm{R}_{50}$ (electrons), symmetry, flatness, etc. ...);

- Specific quality control of each patient on which the VMAT technique is performed;

- Commissioning, (each accelerator has a series of data that will inserted in the planning system);

- Linear Accelerator Acceptance Tests.

For the effects of institutional workload, we considered three photon energies $(6,10 \mathrm{MV}$ and 6MV SRS) and six electrons energies $(4,6,9,12,15,18 \mathrm{MeV})$. The values of MU for commissioning and acceptance were registered in another linear accelerator model Trilogy, with same energies to estimate the MU above mentioned the following procedure was done:

- The beam-on (minutes) time was recorded at the time of the linac arrival at the Américas Oncology Center $\left(\mathrm{t}_{0}\right)$;

- After commissioning and accepting, the beam-on time was noted again $(\mathrm{t})$ :

- The beam-on time $\left(\mathrm{t}-\mathrm{t}_{0}\right)$ was multiplied by the mean dose rate (minutes). Obtained the estimation of MU for linac tests. 


\subsection{VMAT Factor}

Modern radiation therapy techniques increase the MU's when compared to some conventional treatments, particularly those that use no flatness filter. This increase in MU does not significantly affect the thickness of the primary or secondary barrier [1, 17]; however, for leakage radiation, the MUs can increase up to tenfold at institutions that use IMRT [18], and may significantly affect the leakage workload.

NCRP 151 recommends that for obtaining the factor, firstly, one must obtain MU $U_{\text {IMRT }}$, was calculated as shown in equation 1. This protocol recommends values between 2 and 10 .

$$
M U_{I M R T}=\sum \frac{M U_{i}}{\left(D_{\text {pre }}\right)_{i}}
$$

By setting the same dose value, the MU value is obtained, with the following configuration in the linear accelerator: source-surface distance $(\mathrm{SSD})=100 \mathrm{~cm}$; field $=10 \times 10 \mathrm{~cm}^{2}$, and depth $=10$ $\mathrm{cm}, \mathrm{MU}_{\mathrm{conv}}$ is obtained; with the value of this magnitude, the value of the IMRT factor, CI can be obtained, making the following equation 2 :

$$
C_{I}=\frac{M U_{I M R T}}{M U_{c o n v}}
$$

Oliver et al., [7], the number of MU's for modulated volumetric arcs is lower than for the IMRT both in Step-and-Shoot and Sliding Window treatment modalities.

\section{RESULTS AND DISCUSSION}

The total dose for all treatments carried out in the two years of conventional treatment with dose limit up to 3 Gy was $34153 \mathrm{~Gy}$, considering one year, 50 weeks, therefore two years is 100 weeks, so the clinical workload in Gy/week will be:

$$
W_{c}=\frac{34153 \text { Gy }}{100 \text { weeks }} \approx 342^{\text {Gy }} / \text { week }
$$


Similarly, for hypofractionated treatments, we have:

$$
W_{\text {HIPO }}=\frac{3435 G y}{100 \text { weeks }} \approx \mathbf{3 4} \text { Gy } / \text { week }
$$

\subsection{Physical Workload}

The physical workload can be listed according to the institutional dosimetric protocols of all of the quality controls. Total doses of these controls for photon energies of $6 \mathrm{MV}, 6 \mathrm{MV}$ stereotatic radiosurgery (SRS), $10 \mathrm{MV}$ and electrons energies of $6,9,12,15,18 \mathrm{MeV}$ are listed:

- Acceptance $+($ commissioning $\mathrm{x} 4)$; one commissioning every 5 years $=3600+69120$ Gy $=$ $72720 \mathrm{~Gy}$; assuming the total operation time of a 20-year facility (1000 weeks), the workload in Gy/week will be:

$$
\frac{38160}{1000} \approx 38.2 \text { Gy } / \text { week }
$$

- Daily quality control:

Initial Dose Ionization Chamber $500 \mathrm{MU}=5 \mathrm{~Gy}$

Electrons 6 energies $=600 \mathrm{MU}=6 \mathrm{~Gy}$

Arcs clockwise (cw)/counterclockwise $(\mathrm{ccw})=360 \mathrm{MU}=3.6 \mathrm{~Gy}$

Dynamic Wedge $=200 \mathrm{MU}=2 \mathrm{~Gy}$

Total 16.6 Gy x 5 days $\approx 83$ Gy/week

- Monthly absolute dosimetry:

Photons and electrons Initial dose in the ionization chamber; $500 \mathrm{MU}=5 \mathrm{~Gy}$

Total photons energies $6000 \mathrm{MU}=60 \mathrm{~Gy}$

Total electrons energies $9000 \mathrm{MU}=90 \mathrm{~Gy}$

Total $155 \mathrm{~Gy} /$ month $\approx 40 \mathrm{G} \boldsymbol{/} /$ week

- Quarterly tests 2 Gy $/$ week

- Annual quality control: Photons. 
Photons $6 \mathrm{MV}, 10 \mathrm{MV}$ : $100 \mathrm{~Gy} /$ year, for the case of this facility, there are energies of 6 and 10 MV. For measurements of 6MV SRS photons, measurement of the filter factor is not done, so the institutional value is 75 Gy/year.

- Annual quality control: Electrons [16].

The energies of existing institutional electrons are 4, 6, 9, 12, 15, 18, $21 \mathrm{MeV}$. The dose value per energy performed by the annual test is 90 Gy/year. Thus, the total dose released in the annual tests will be:

$((100 x 2)+75+(90 \times 6))=815$ Gy/year

Weekly, the annual tests would have the following value

$$
\frac{815}{50}=16.3^{G y} / \text { week }
$$

- Patient specific quality control (40 patients) $\approx 23.3$ Gy/ week

Thus, the dose in Gy/week of the physical workload will be:

$\mathrm{W}_{\mathrm{f}}=38.2+83+40+2+16.3+23.3 ; \mathrm{W}_{\mathrm{f}} \approx 203$ Gy/week.

The total workload will be:

$\mathrm{W}_{\mathrm{T}}=\mathrm{W}_{\mathrm{C}}+\mathrm{W}_{\mathrm{F}} ; \mathrm{W}_{\mathrm{T}}=342+34+203 \approx 579$ Gy/week.

Generally, the number of energies in accelerator determines the physical workload value, but in facilities that realize VMAT treatments, the total of patients treated is important too, so table 1 presents the specific patient quality control for the different number of patients.

In the analyzed data of all the treated patients, it was possible to obtain an estimate of the total dose for VMAT treatments for the procedure of specific quality control of patients for services that have 40, 80 and 120 patients, these values are shown in table 1.

Table 2 shows the institutional workload values, of the references [19, 20] and this study. The results show that the actual workload value is less than half of the facility design that was submitted to the regulatory agency, CNEN (Comissão Nacional de Energia Nuclear) [21].

Table 1: Suggested patient specific quality control, values for exclusive VMAT treatments. 


\begin{tabular}{cc}
\hline Number of Patients & Workload (Gy/week) \\
\hline Up to 40 patients & 23.3 \\
Up to 80 patients & 47 \\
Up to 120 patients & 70 \\
\hline
\end{tabular}

Table 2: comparing different standard workloads and this study.

\begin{tabular}{cc}
\hline Reference & Workload (Gy/week) \\
\hline Institutional Shielding Design & 1250 \\
Kleck and Elsalim 1994 [19] & 600 \\
Mechalakos 2004 [20] & 850 \\
This Study & 579 \\
\hline
\end{tabular}

Table 3 presents the suggestion for workload by the authors, according to the number of patients treated with the VMAT technique.

Table 3: workload values suggested by authors for exclusive VMAT treatments per patients.

\begin{tabular}{cc}
\hline Number of Patients & Workload (Gy/week) \\
\hline Up to 40 patients & 600 \\
Up to 80 patients & 1000 \\
Up to 120 patients & 1500 \\
\hline
\end{tabular}

The VMAT factor is obtained first by calculating the MUVMAT, which is obtained using equation 1:

$$
M U_{I M R T}=\frac{482567}{168002} \approx 2.9
$$

Then $\mathrm{MU}_{\text {conv }}$ is obtained:

$$
M U_{\text {conv }}=\frac{251587}{168002} \approx 1.5
$$


Next, one obtains the VMAT $C_{I}$ factor for conventional treatments will be:

$$
C_{I}=\frac{2.9}{1.5} \approx 1.93
$$

Similarly, the VMAT factor for hypofractionation $\mathrm{C}_{2}$ will be:

$$
C_{2}=\frac{2.31}{1.50} \approx 1.54
$$

The VMAT factor results per site are shown in Table 4.

Table 4: VMAT factor per anatomical region.

\begin{tabular}{cc}
\hline Anatomical Region & VMAT Factor \\
\hline Prostate (men) & 2.1 \\
Breast (women) & 1.2 \\
Colon and Rectum (both) & 1.5 \\
Trachea, Bronchus and Lung (both) & 1.5 \\
Stomach (both) & 1.5 \\
Cervix (women) & 1.8 \\
Oral Cavity(both) & 1.6 \\
\hline
\end{tabular}

Modern radiotherapy techniques, such as VMAT, results in drastic modifications in workload calculation. This work showed that the new quality controls specified in the TG-142 [14], had as consequence the increase of physical workload, these controls have dependences mainly for energies, however, differently from 3D-CRT, in VMAT treatments the number of patients is an important parameter to be available, having the effect in the increase of the physical workload too, so in Table 1 was shown that the total of the patient-specific quality control treated with VMAT technique, these values lead to a more accurate analysis of the physical workload.

Table 2 showed that total workload is lower than the value that was submitted to regulatory agency Comissão Nacional de Energia Nuclear (CNEN), however, this workload was obtained 
considering 40 patients per day, that is below of Brazilian reality. Table 3 gives different values of total workload, so the evaluation of total patients treated will determine the most appropriate choice.

The VMAT factor results demonstrate that two considerations do not result in significant differences, the first is separate the traditional treatments of the hypofractionation, the second is the average value calculated for all treatments, 1.93, and the values per site.

The authors recommend that facilities performing exclusively VMAT treatments use the overestimated value to IMRT factor of 2.5 to calculate leakage shielding. This is slightly higher than the lower threshold values recommended by NCRP 151, which are between 2 and 10 for IMRT factors and half of the value recommended by the national regulatory agency Comissão Nacional de Energia Nuclear (CNEN) [21].

\section{CONCLUSIONS}

Generally, the standard references in radiotherapy shielding show the total workload [8,9], however, this work recommends that the workloads, clinical and physical are calculated separately, this demonstration is more reliable for the calculation of thickness of bunkers in facilities that perform VMAT treatments.

Radiation therapy services are migrating to high technology with arc treatments, and it was shown that important values in the calculation of shielding - such as physical workload, clinical workload, MUIMRT and VMAT factor need to be constantly updated. But conventional treatments continue to be used for palliative treatments and conformal therapy as well, especially in our reality. 


\section{ACKNOWLEDGMENT}

The authors thank the Clínicas Oncológicas Integradas and the Federal University of Rio de Janeiro.

\section{REFERENCES}

[1] RODGERS, J. E.; Radiation therapy vault shielding calculational methods when IMRT and TBI procedures contribute. J Appl Clin Med Phys, v. 2(3), p. 157-164, 2001.

[2] FOLLOWILL, D., GEIS, P., and BOYER, A. Estimates of whole-body dose equivalent produced by beam intensity modulated conformal therapy. Int J Radiat Oncol Biol Phys, v. 38, p. 667-672, 1997.

[3] YU, C. X. Intensity-modulated arc therapy with dynamic multileaf collimation: An alternative to tomotherapy. Phys Med Biol, v. 40, p. 1435-1449, 1995.

[4] CROOKS, S. M., WU, X., TAKITA, C., WATZICH, M., and XING, L., Aperture modulated arc therapy. Phys Med Biol, v. 48, p. 1333-1344, 2003.

[5] BRATENGEIER, K., 2-Step IMAT and 2-Step IMRT in three dimensions Med Phys, v. 32, p. 3849-3861, 2005.

[6] OLIVER, M., ANSBACHER, W., BECKHAM, W. A. Comparing planning time, delivery time and plan quality for IMRT, Rapidarc and tomotherapy. J Appl Clin Med Phys, v. 10, p. 117$131,2009$.

[7] OTTO K. Volumetric modulated arc therapy: IMRT in a single gantry arc. Med Phys, v. 35(1), p. $310-17,2008$.

[8] IAEA - International Atomic Energy Agency. Radiation Protection in the Design of Radiotherapy Facilities. Safety Report Series, IAEA, Vienna 2006. 
[9] NCRP - National Council on Radiation Protection and Measurements. Structural Shielding Design and Evaluation for Megavoltage $X$ and Gama-Ray Radiotherapy Facilities. NCRP Report No. 151 Bethesda, MD: NCRP, 2005.

[10] BLOMGREN H. et al. Stereotactic high dose fraction radiation therapy of extracranial tumors using an accelerator. Acta Oncol, v. 34, p. 861-870, 1995.

[11] MCGARRY, R. C., PAPIEZ, L. and WILLIAMS M. Stereotactic body radiation therapy of early-stage non-small-cell lung carcinoma: Phase I study. Int J Radiat Oncol Biol Phys, v. 63, p. 1010-1015, 2005.

[12] BENEDICT, S. H. et al. Stereotactic body radiation therapy: The report of AAPM Task Group 101 of the Therapy Physics Committee of AAPM, Med Phys, v. 37(8), p. 4078-4101, 2010.

[13] DAS, I. et al. Accelerator beam data commissioning equipment and procedures: Report of the TG-106 of the Therapy Physics Committee of the AAPM. Med Phys, v. 35, p. 4186-4215, 2008.

[14] KLEIN, E. E. et al. Quality assurance of medical accelerators: Report of the TG-142 of the Therapy Physics Committee of the AAPM. Med Phys, v. 36, p. 4197- 4212, 2009.

[15] IAEA - International Atomic Energy Agency. Absorbed Dose Determination in External Beam RadioTherapy. Technical Reports Series No. 398, IAEA, Vienna, 2000.

[16] CAMPOS, L. T.; Da ROSA, L. R.; BRAZ, D. Quality Control of Radiotherapy treatment plans with electrons. Braz J Rad Sci, v. 7, p. 1-21, 2019.

[17] MUTIC, S. and LOW, D.A. Whole-Body dose from tomotherapy delivery. Int J Radiat Oncol Biol Phys, v. 42(1), p. 229-232, 1998.

[18] PURDY, J.A. et al. Intensity Modulated Therapy: Current status issues of interest. Int J Radiat Oncol Biol Phys, v. 51(4), p. 880-914, 2001.

[19] KLECK, J.H. and ELSALIM, M. Clinical workloads and use factors for medical linear accelerators. (abstract) Med Phys 21, 952-953, 1994. 
[20] MECHALAKOS, J., ST. GERMAIN, J. and BURMAN, C.M. Results of a one year survey of output for linear accelerators using IMRT and non-IMRT techniques J Appl Clin Med Phys 5(1), 64-72, 2004.

[21] CNEN - Comissão Nacional de Energia Nuclear. Diretrizes básicas de proteção radiológica. D.O.U.: Nov. 14, 2005. (CNEN-NN-3.01). 